\title{
A novel polysaccharide from Se-enriched Ganoderma lucidum induces apoptosis of human breast cancer cells
}

\author{
DEJING SHANG, YANG LI, CHE WANG, XIAOMIN WANG, ZHI YU and XIN FU \\ Liaoning Provincial Key Laboratory of Biotechnology and Drug Discovery, College of Life Science, \\ Liaoning Normal University, Dalian 116029, P.R. China
}

Received August 2, 2010; Accepted September 14, 2010

DOI: $10.3892 /$ or_00001070

\begin{abstract}
The novel polysaccharide SeGLP-2B-1 isolated from Se-enriched Ganoderma lucidum, showed antiproliferative activity towards several cancer cell lines in vitro. To investigate the antitumor mechanisms, the apoptotic effects of SeGLP-2B-1 in human breast cancer cells were studied, and the mechanism of this action was further elucidated. Cell apoptosis was detected by Annexin V/PI staining. Caspase activity was assayed using a caspase apoptosis detection kit. Western blot analysis was used to evaluate the levels of procaspase-3, -8, -9, PARP and cytochrome $c$ expression. The results showed that SeGLP-2B-1 inhibited the growth of MCF-7 cells in a time- and dose-dependent manner. Typical characteristics of apoptosis were observed, including morphological changes, sub-G1 cells and DNA ladder formation. Further analysis showed that SeGLP-2B-1 treatment disrupted the mitochondrial membrane potential followed by an increase in the cytochrome $c$ cytosolic levels. Sequentially, SeGLP2B-1 increased the activities of caspase-9, -3 and poly (ADPribose) polymerase in a time-dependent manner, however, no obvious activation of caspase- 8 was observed. Caspase- 9 and caspase-3 inhibitor prevented SeGLP-2B-1-induced apoptosis, and the activities of caspases-3, -9 were significantly upregulated by SeGLP-2B-1. Our studies suggest that SeGLP2B-1 induces apoptosis via a mitochondria-mediated pathway.
\end{abstract}

\section{Introduction}

Epidemiological studies suggest that dietary and chemoprevention can provide an important and potentially feasible pathway for reducing cancer occurrence. Selenium (Se) is an essential trace element for a number of metabolically important enzymes (1). Accumulating evidence indicates that a signi-

Correspondence to: Dr Dejing Shang, Liaoning Provincial Key Laboratory of Biotechnology and Drug Discovery, College of Life Science, Liaoning Normal University, Dalian 116029, P.R. China E-mail: djshang@lnnu.edu.cn

Key words: chemoprevention, Se-containing polysaccharide, Ganoderma lucidum, antiproliferative, apoptosis, mitochondria ficant inverse relationship between intake of Se and cancer incidence and $\mathrm{Se}$ has received considerable attention for its potential role as a chemopreventive agent $(2,3)$. Selenite induce cell necrosis accompanied by damage to cellular DNA and loss of cell-membrane integrity $(4,5)$. Organic selenocompounds induced apoptosis (programmed cell death) without producing changes in membrane integrity or cellular DNA as measured by single-strand breaks in DNA (6-8). Both organic and inorganic compounds of Se have been demonstrated to manifest cancer preventive potential, but several organic seleno-compounds have shown higher anticancer activities, lower toxicity and fewer side effects than selenite, therefore, it is crucial to develop suitable organic Se sources for cancer chemoprevention.

Se-containing polysaccharide, a nutritionally available organic seleno-compound, has been shown to promote cancer cell differentiation, suppress cell division and induce programmed cell death by regulating cancer gene expression $(9,10)$. Although several mechanisms have been proposed to account for anticancer effects of Se-containing polysaccharide, selective induction of tumor cell apoptosis may be of particular significance as chemopreventive agents (11-14).

SeGLP-2B-1, a Se-containing polysaccharide, was purified and characterized from the Se-enriched Ganoderma lucidum for the first time in our laboratory. Previous studies demonstrated that SeGLP-2B-1 exhibited strong antiproliferative activity against six human cancer cell lines in a Se-dependent manner (15). Treatment of cancer cells with SeGLP-2B-1 produced the typical characteristics of apoptosis, including cell shrinkage, compaction of cytoplasmic organelles, karyopyknosis, condensation and margination of nuclear chromatin, and cytoplasmic vacuolization. In our current study, we investigated the effects of SeGLP-2B-1 on cell growth inhibition and apoptosis in the breast cancer cell line MCF-7. We show that SeGLP-2B-1 indeed decreases cell viability and induces apoptosis. Our results also demonstrate that SeGLP-2B-1 induces MCF-7 cell apoptosis via a mitochondrial pathway.

\section{Materials and methods}

Preparation of SeGLP-2B-1. SeGLP-2B-1 was prepared as described (15). SeGLP-2B-1 was isolated and identified from the mycelia of Se-enriched G. lucidum using a DEAE-52 column, a TSK-G5000PW HPLC column and a SHIM- 
PACK HPLC column. The polysaccharide content was determined with the phenol-sulfate method using glucose as the standard (16). The molecular weight of SeGLP-2B-1 was determined by HPSEC. The Se content of SeGLP-2B-1 was evaluated using inductively coupled plasma-atomic emission spectroscopy (Plasma-Spec-II, Leeman Labs) as reported (17). Standard bovine liver powder was the normal control.

Cell viability assay. Cell viability was assessed using the MTT assay (18). Briefly, MCF-7 cells were incubated in RPMI-1640 with $10 \%$ (v/v) heat-inactivated fetal calf serum in 96-well plates. After a 24-h incubation, SeGLP-2B-1 was added to each well to a final concentration ranging from 0.045 to $0.36 \mu \mathrm{M}$ for 24,48 or $72 \mathrm{~h}$. At the end of the treatment, $20 \mu \mathrm{l}$ of MTT solution was added to each well and incubated for an additional $4 \mathrm{~h}$. After washing and centrifugation, $150 \mu \mathrm{l}$ of DMSO was added to dissolve the MTT formazan crystals. The spectrophotometric absorbance at $592 \mathrm{~nm}$ was determined using a scanning multiwell spectrophotometer. In each experiment, the MTT assay was performed in eight replicates. Each experiment was repeated three times.

Annexin V/PI staining. Cells treated with $0.18 \mu \mathrm{M}$ SeGLP2B-1 were harvested and labeled with Annexin V/PI conjugated to fluorescein (Roche Diagnostics, Indianapolis, IN). The labeled cells were analyzed by flow cytometry on an EPICS ${ }^{\circledR}$ XL flow cytometer (Beckman Coulter, Fullerton, CA).

Cell cycle analysis. Cells incubated with $0.18 \mu \mathrm{M}$ SeGLP2B-1 were collected, washed, suspended in ice-cold PBS, gently fixed in $70 \%$ ice-cold ethanol at $-20^{\circ} \mathrm{C}$ for $24 \mathrm{~h}$ and stained with PI in a dark room at room temperature for $30 \mathrm{~min}$. Cell cycle analysis was performed using an EPICS ${ }^{\circledR} \mathrm{XL}$ flow cytometer, and the results were analyzed by the system-III software (Beckman Coulter). The proportion of sub-G1 hypodiploid cells was quantitated and presented as a percentage of apoptotic cells. The results are presented as the mean \pm SD from three independent experiments.

Mitochondrial membrane potential $(\Delta \Psi m)$ assay. Cells treated with $0.18 \mu \mathrm{M}$ SeGLP-2B-1 were stained with rhodamine123 and analyzed using a FACSCalibur flow cytometer with an excitation wavelength of $488 \mathrm{~nm}$ and an emission wavelength at $525 \mathrm{~nm}$ for green fluorescence. The mean fluorescence intensity (MFI) from rhodamine123 was used to indicate the $\Delta \Psi \mathrm{m}$ of cells.

Western blot analysis. Cells treated with $0.18 \mu \mathrm{M}$ SeGLP2B-1 were washed and then lysed in $50 \mathrm{mM}$ Tris- $\mathrm{HCl}, \mathrm{pH} 8.0$, containing $1 \mathrm{mM}$ PMSF, $1 \mathrm{mM}$ EDTA, $150 \mathrm{mM} \mathrm{NaCl}$ and $1 \%$ SDS. Cell lysates were obtained by centrifugation at $10000 \mathrm{x} \mathrm{g}$ for $40 \mathrm{~min}$ at $4^{\circ} \mathrm{C}$, and the protein content of the supernatant was determined using the Bradford method (19). Proteins were subjected to electrophoresis on $13.3 \%$ SDSpolyacrylamide gels and were subsequently transferred to a polyvinyl difluoride membrane. Membrane was blocked with non-fat milk in PBS containing $0.1 \%$ Tween-20, and then treated with primary antibodies overnight at $4^{\circ} \mathrm{C}$ and horseradish peroxidase-conjugated anti-rabbit $\mathrm{IgG}$ or anti-mouse

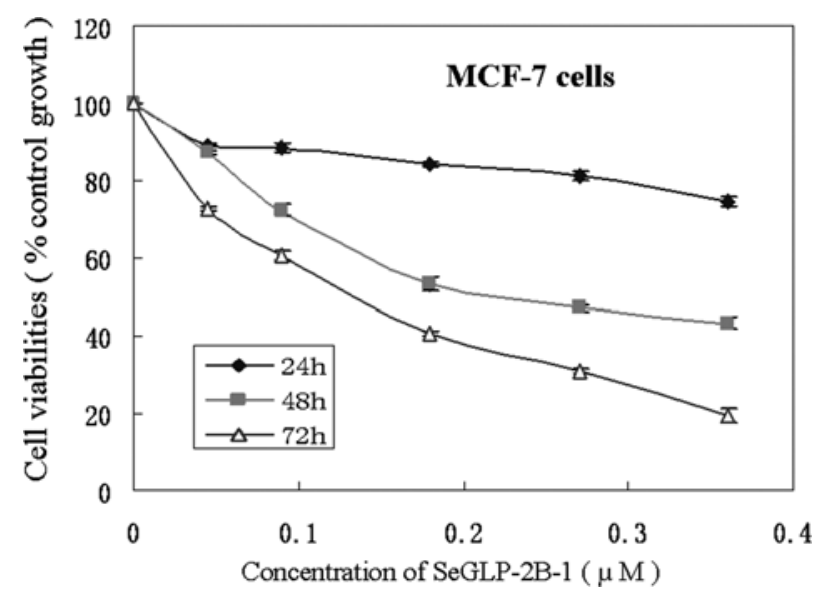

Figure 1. Effect of SeGLP-2B-1 on MCF-7 cell viability. MCF-7 cells were treated with SeGLP-2B-1 at concentrations from 0 to $0.36 \mu \mathrm{M}$ for 24,48 or $72 \mathrm{~h}$. Cell viability assays were performed as described in Materials and methods. Results shown represent the mean $\pm \operatorname{SD}(n=3)$.

$\operatorname{IgG}(1: 5000)$ for $1 \mathrm{~h}$ at $30^{\circ} \mathrm{C}$. Immunoreactive bands were visualized using the ECL Western Blotting Detection system kit (Amersham Biosciences, Buckinghamshire, UK). B-actin was detected as a control. The intensities of the protein bands were quantitated with densitometry using Scion Image software.

Caspase activity and effect of caspase inhibitors. Cells were treated with $0.18 \mu \mathrm{M}$ SeGLP-2B-1 after preincubation with caspase inhibitor (caspase-3 inhibitor, z-DEVD-FMK; caspase-8 inhibitor, z-IETD-FMK; caspase-9 inhibitor, zLEHD-FMK) for $1 \mathrm{~h}$. Cell viability was evaluated using the MTT method. Caspase-3, -8 and -9 activities were measured using a Caspase Apoptosis Detection kit (Biovision Inc. Mountain View, CA).

Statistical analysis. The data were analyzed using one-way analysis of variance and Duncan's test. Triplicate samples were used to generate each data point. Statistical significance was set at $\mathrm{p} \leq 0.05$.

\section{Results}

SeGLP-2B-1 reduces the viability of $M C F-7$ cells. The effect of SeGLP-2B-1 on MCF-7 cell viability was examined after cells were treated with increasing concentrations of SeGLP2B-1 for 24-72 h. At $24 \mathrm{~h}$, the cell viability of MCF-7 cells treated with SeGLP-2B-1 at various concentrations was not significantly decreased (Fig. 1). After 48 h, MCF-7 cell viability was significantly reduced in a dose-dependent manner; at $0.18 \mu \mathrm{M}$ SeGLP-2B-1, the number of survival cells decreased by $>50 \%$.

SeGLP-2B-1 induces apoptosis in MCF-7 cells. To investigate whether the observed SeGLP-2B-1-induced decrease in MCF-7 cell viability was due to apoptosis, firstly, we assessed the formation of sub-G1 apoptotic bodies. The results showed that the proportion of sub-G1-phase cells increased over time (Fig. 2). Before treatment with $0.18 \mu \mathrm{M} \mathrm{SeGLP-2B}-1$ for $24 \mathrm{~h}$, 


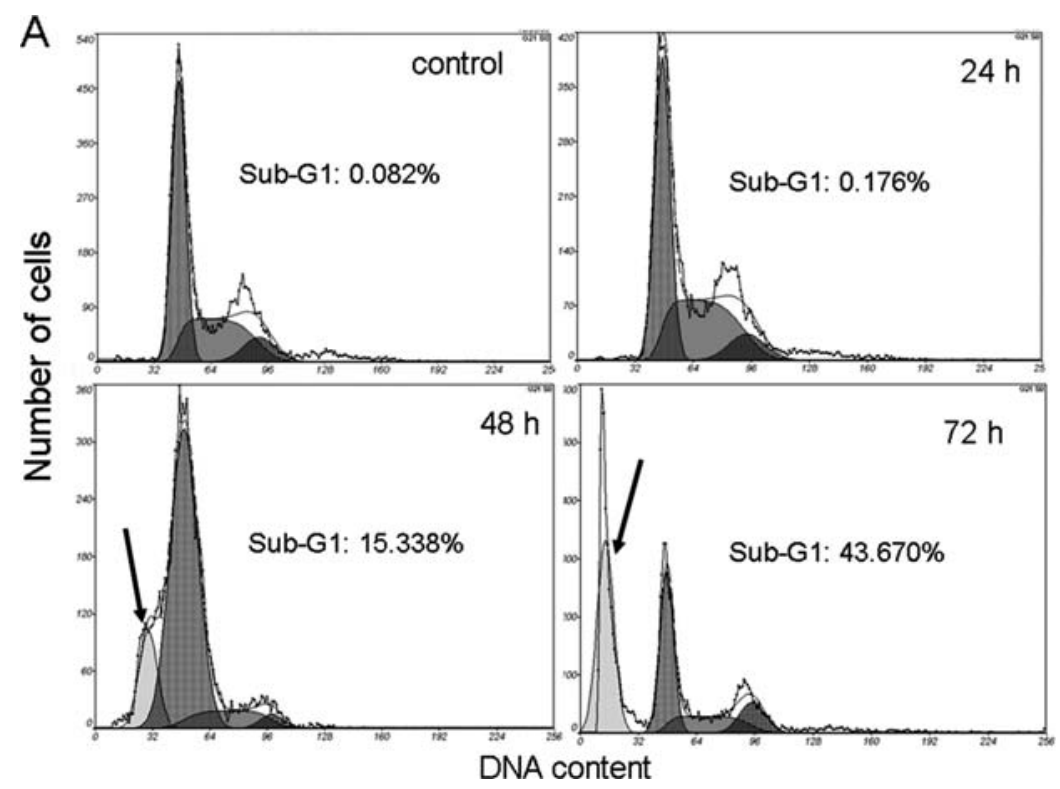

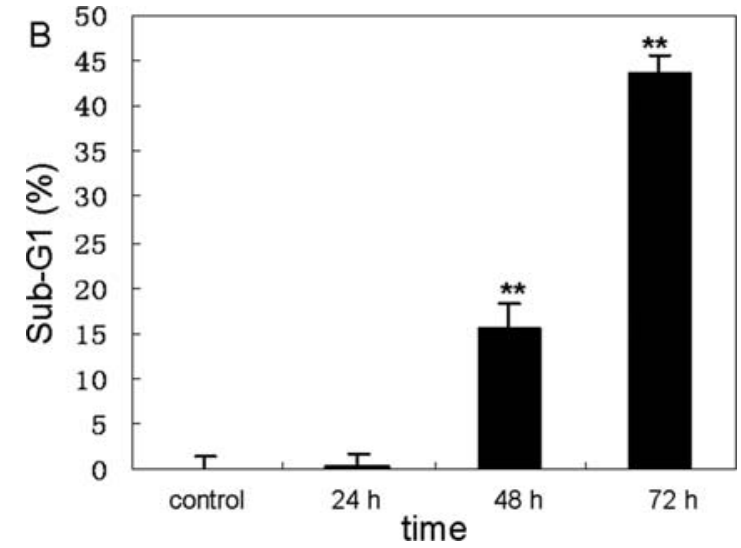

Figure 2. The formation of sub-G1 apoptotic bodies in MCF-7 cells. (A) MCF-7 cells treated with SeGLP-2B-1 were collected, fixed and stained with PI. The cell cycle phase distribution was analyzed using flow cytometry. The arrow indicates the sub-G0/G1 phase (apoptotic cell peak). (B) Quantitative analysis of the proportion of cells in the sub-G1 phase. Each value represents the mean of three independent experiments. ${ }^{* *} \mathrm{p} \leq 0.01 \mathrm{com}-$ pared with control group.

no obvious sub-G1 peak (\% apoptotic cells) was observed, but after incubation with SeGLP-2B-1 the proportion of apoptotic cells increased significantly $(\mathrm{p} \leq 0.05)$ from $0.082 \%$ (control) to $15.338 \%(48 \mathrm{~h})$ and $43.670 \%(72 \mathrm{~h})$. Analysis using Annexin V/PI staining further confirmed that SeGLP2B-1 induced apoptosis. As shown in Fig. 3, few apoptotic cells were observed before treatment with $0.18 \mu \mathrm{M}$ SeGLP2B-1 for $24 \mathrm{~h}$, but early apoptotic cells and secondary necrotic cells were present at 48 and $72 \mathrm{~h}$, respectively.

Mitochondria dysfunction induced by $S e G L P-2 B-1$. A variety of key events in apoptosis involve mitochondria, including loss of $\Delta \Psi \mathrm{m}$ and the release of caspase activators such as cytochrome $c$. The disruption of mitochondrial integrity is one of the early events leading to apoptosis. To examine if mitochondria play a role in the observed SeGLP-2B-1-induced apoptosis, we first measured $\Delta \Psi \mathrm{m}$ in MCF-7 cells treated with SeGLP-2B-1 using flow cytometry after rhodamine123
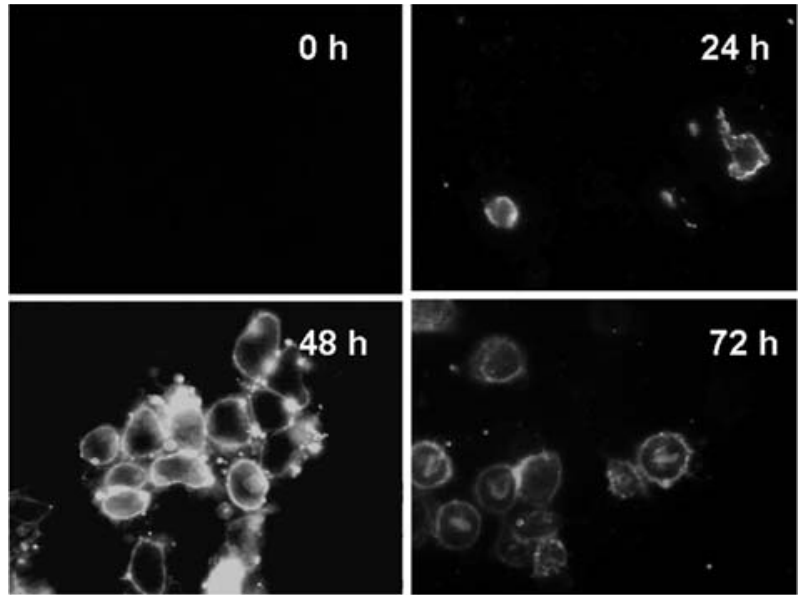

Figure 3. SeGLP-2B-1-induced apoptosis of MCF-7 cells. $\mathrm{AV}^{+} / \mathrm{PI}^{-}$cells represent early apoptotic cells, and $\mathrm{AV}^{+} / \mathrm{PI}^{+}$represent secondary necrotic cells.

staining. As shown in Table I, the change of $\Delta \Psi \mathrm{m}$ was observed in treated MCF-7 cells. Slight decrease of mean fluorescence intensity was detected in cells treated with $0.18 \mu \mathrm{M}$ SeGLP-2B-1 at $24 \mathrm{~h}$. Compared to control, the decrease in the mean fluorescence density was about 37.9$54.3 \%$ at 48 and $72 \mathrm{~h}$, respectively. The results showed that SeGLP-2B-1 could induce the dissipation of mitochondrial $\Delta \Psi \mathrm{m}$ in a time-dependent manner.

The release of cytochrome $c$. A reduction in the mitochondrial membrane potential is usually accompanied by release of cytochrome $c$ into the cytosol. The release of cytochrome $c$ from mitochondria is a particularly important event in the induction of apoptosis. Therefore, we examined the amount of cytochrome $c$ in the cytoplasm using Western blotting. Cyto-chrome $c$ was released from mitochondria, which resulted in a significant $(\mathrm{p} \leq 0.05)$ increase in cytosolic cytochrome $c$. No significant increase was seen within $24 \mathrm{~h}$. 
Table I. The disruption of $\triangle \Psi \mathrm{m}$ in MCF-7 cells treated with SeGLP-2B-1 for 0-72 h.

\begin{tabular}{lcc}
\hline & $\begin{array}{c}\text { Mean fluorescence } \\
\text { intensity (MFI) }\end{array}$ & $\begin{array}{c}\text { Lost } \Delta \Psi \mathrm{m} \\
(\% \text { control })\end{array}$ \\
\hline Control & $225.2 \pm 63.4$ & \\
$24 \mathrm{~h}$ & $208.8 \pm 13.5$ & $7.2 \pm 3.2$ \\
$48 \mathrm{~h}$ & $119.7 \pm 56.2^{\mathrm{a}}$ & $46.8 \pm 16.8^{\mathrm{a}}$ \\
$72 \mathrm{~h}$ & $103.6 \pm 37.5^{\mathrm{a}}$ & $54.3 \pm 9.2^{\mathrm{a}}$ \\
\hline
\end{tabular}

Reported values are presented as the mean $\pm \mathrm{SD}(\mathrm{n}=3) .{ }^{\mathrm{a}} \mathrm{p}<0.05$ is significantly different from that of the control.

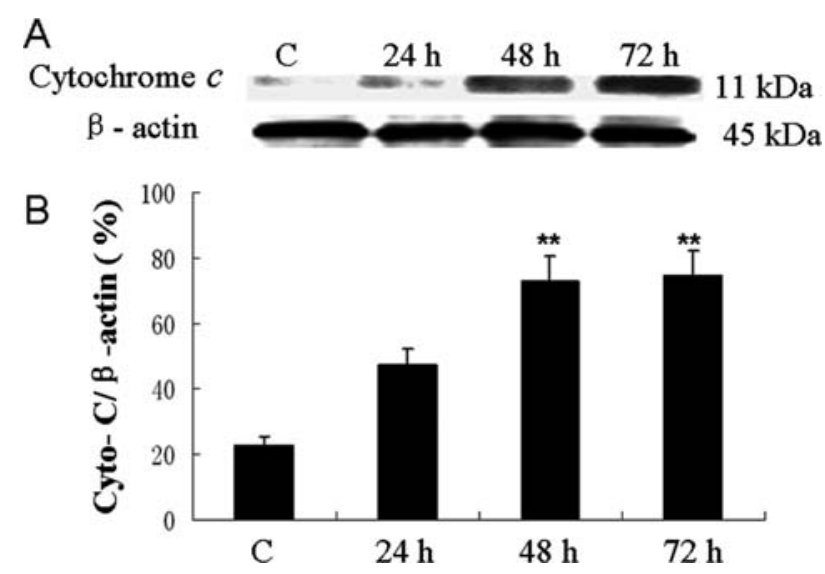

Figure 4. (A) Western blot analysis of the expression of cytochrome $c$ in the cytoplasm. (B) Relative intensities of the protein bands were quantified by densitometry and were scaled to the $B$-actin reference bands. Values are the means $\pm \operatorname{SD}(\mathrm{n}=3) .{ }^{* *} \mathrm{p} \leq 0.01$ compared with the control.

After $48 \mathrm{~h}$, about 3-fold increase in the cytochrome $c$ level was observed (Fig. 4). These results indicated that SeGLP2B-1 induced apoptotic cell death via a mitochondriamediated pathway.

Effect of caspase on SeGLP-2B-1-induced apoptosis in MCF-7 cells. Release of cytochrome $c$ from mitochondria can trigger a series of events leading to the activation of effector caspases. To investigate the role of caspases in SeGLP-2B-1-induced cell apoptosis, the effects of SeGLP-2B-1 on the expression of caspase-8, caspase-9, caspase-3 and PARP in MCF-7 cells were determined by Western blotting. SeGLP-2B-1 induced the degradation of procaspase- 8 , procaspase- 9 and procaspase-3 and PARP cleavage in a time-dependent manner (Fig. 5). PARP was cut into small fragments of $83 \mathrm{kDa}$ by activated caspase-3, and with time, the expression of $83 \mathrm{kDa}-$ specific fragment gradually increased. To further examine the participation of caspases in SeGLP-2B-1-induced apoptosis, we used the specific fluorogenic peptide substrates IETDpNA, LEHD-pNA and DEVD-pNA to detect caspase-8, caspase- 9 and caspase- 3 activities, respectively. The results indicated that caspase activity was significantly increased in a time-dependent manner after the cells were treated with

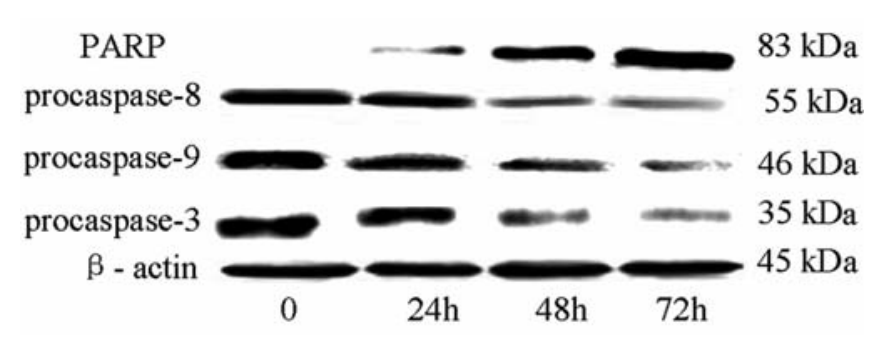

Figure 5. Western blotting of procaspase-8, -9, -3 and PARP expressed in MCF-7 cells treated with SeGLP-2B-1.

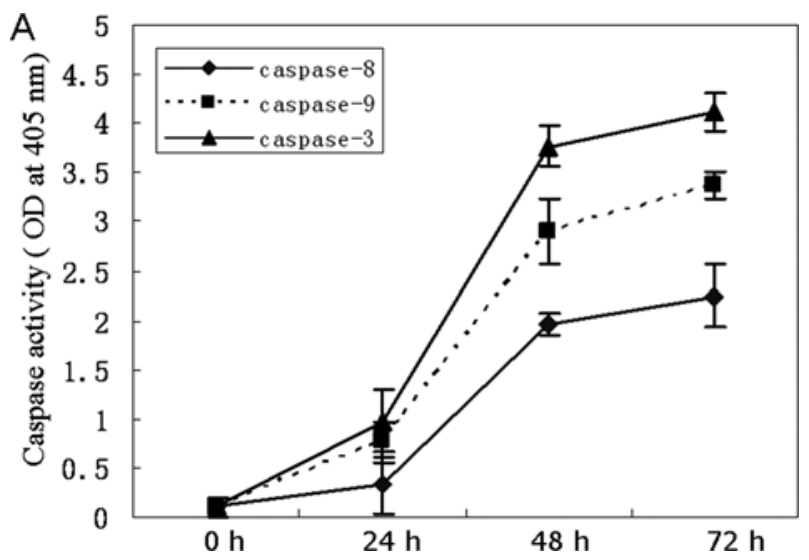

B

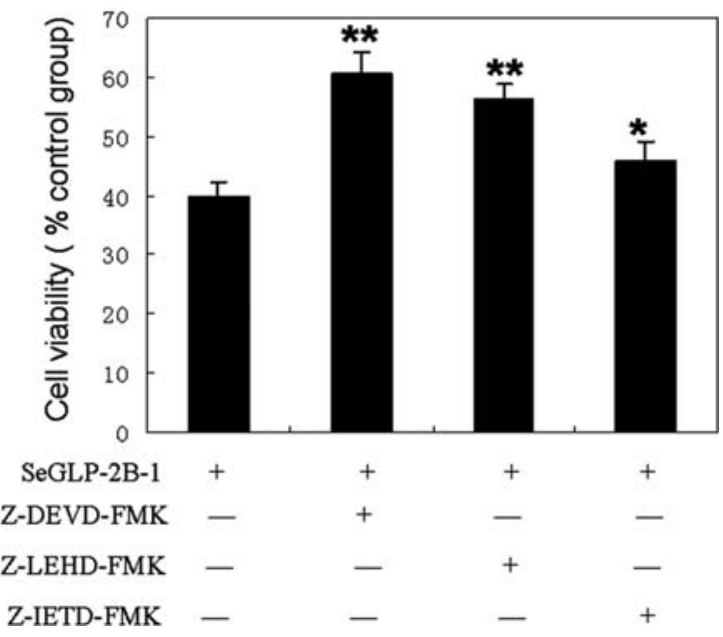

Figure 6. Effect of caspases on apoptosis induced by SeGLP-2B-1 in MCF-7 cells. (A) Activities of caspase-3, -8 and -9 of MCF-7 cells treated with SeGLP-2B-1. (B) Effect of caspase inhibitors on SeGLP-2B-1-induced apoptosis in MCF-7 cells. The cells were cultured in the absence or presence of caspase inhibitors. At $2 \mathrm{~h}$ prior to the addition of SeGLP-2B-1, caspase inhibitors were added and the cells were further incubated for $72 \mathrm{~h}$. Data shown are the means $\pm \mathrm{SD} n=3 ;{ }^{*} \mathrm{p} \leq 0.05,{ }^{* *} \mathrm{p} \leq 0.01$ compared with the SeGLP-2B-1 group.

$0.18 \mu \mathrm{M}$ SeGLP-2B-1 (Fig. 6A). We examined cell viability when cells were treated with SeGLP-2B-1 in the absence or presence of the caspase-3 inhibitor, z-DEVD-FMK or the caspase-9 inhibitor, z-LEHD-FMK, or the caspase-8 inhibitor, z-IETD-FMK, which were used to block intracellular proteases. Treatment with caspase $-3,-8$ or -9 inhibitor increased the 
number of viable MCF-7 cells in the presence of SeGLP-2B-1 (Fig. 6B). Cell viabilities in the presence of no inhibitor, caspase- 3 inhibitor, caspase- 8 inhibitor and caspase- 9 inhibitor were $39.7,60.6,46.0$ and $56.3 \%$, respectively. These results indicated that both caspase- 3 and caspase- 9 inhibitors significantly increased cell viability in the presence of SeGLP-2B-1 in MCF-7 cells ( $\mathrm{p} \leq 0.01)$, and also caspase-8 inhibitors increased cell viability $(\mathrm{p} \leq 0.05)$.

\section{Discussion}

The goal of cancer chemoprevention is to inhibit the proliferation of cancer cells and to selectively induce cancer cell death using natural or synthetic compounds. Further understanding of the chemotherapeutic mechanisms of candidate compounds may provide a rational approach to using such agents to enhance apoptosis as a strategy for effective chemoprevention of cancer (20). Accumulating evidence supports the hypothesis that apoptosis is a critical mechanism in cancer chemoprevention by Se-containing compounds (21). The synthetic Se-enriched chemotherapeutic polysaccharide $\kappa$-selenocarrageenan might inhibit proliferation of K562/ADM cells and induce apoptosis via a Fascaspase-3 pathway (22). A Se-protein polysaccharide obtained by water extraction of Se-enriched Agaricus blazei inhibited K562 cell proliferation and caused morphological changes characteristic of apoptosis, nuclear DNA fragmentation, and an increased in caspase-3 activity in vitro, indicating that this Se-protein polysaccharide induced apoptosis of K562 cells via a caspase-dependent apoptotic pathway (23). Our present data demonstrated that SeGLP-2B-1 decreased MCF-7 cell viability via apoptosis.

The apoptotic signal is propagated by either of two distinct mechanisms: the extrinsic pathway or the intrinsic mitochondrial apoptosis pathway (24). The extrinsic pathway involves caspase- 8 activation, which then directly activates the downstream effector caspase- $3,-6$ and -7 , leading to apoptosis. The intrinsic pathway depends on mitochondria, in which caspase- 8 activation results in the proteolysis of the Bcl-2 interacting domain, causing translocation of the smaller Bcl-2 product to mitochondria where it induces the mitochondrial apoptosis pathway (25). The subsequent disruption of $\Delta \Psi \mathrm{m}$ and release of proapoptotic molecules such as cytochrome $c$ and Smac/DIABLO result in caspase-9/caspase-3 activation and apoptosis (26).

Our current study showed that caspase- 8 was involved in SeGLP-2B-1-induced apoptosis as shown by an increase in caspase- 8 activity and down-regulation of procaspase- 8 expression. Down-regulation of procaspase- 8 expression did not significantly reduce cell viability, however, suggesting that caspase- 8 does not directly regulate the proteolysis of caspases during SeGLP-2B-1-induced apoptosis. On the contrary, SeGLP-2B-1 induced both time-dependent degradation of procaspase- 9 and -3 and PARP cleavage, suggesting that caspase- 8 activation may involve the mitochondrial pathway. Both the caspase-3 (z-DEVD-FMK) and caspase-9 (z-LEHD-FMK) inhibitors significantly blocked intracellular proteases, resulting in increased cell viability.

Disruption of $\Delta \Psi \mathrm{m}$ and release of cytochrome $c$ are critical events in both the caspase-dependent and -independent apoptotic pathways (27). In our study, $\Delta \Psi \mathrm{m}$ paralleled the apoptosis data and was consistent with the hypothesis that SeGLP-2B-1 induced mitochondria-mediated cell death. The results suggested that SeGLP-2B-1 caused early damage to mitochondrial membrane potential by forming pores in the mitochondrial membrane known as permeability transition pores through which a proportion of apoptotic factors were released. Release of cytochrome $c$ from the mitochondria into the cytoplasm serves as a trigger to activate caspase- 9 . Inhibitors of caspase-3, -8 and -9 partially blocked SeGLP2B-1-induced MCF-7 cell apoptosis. At the same time, there was an increase in caspase-3, -8 and -9 activities and PARP cleavage, indicating that caspases participated in this apoptotic process. Our results provide a potential molecular mechanism for SeGLP-2B-1-induced apoptosis of MCF-7 cells by the mitochondria-mediated pathways.

\section{Acknowledgements}

The study was supported by the National Natural Science Foundation of China (Grant no. 30770276).

\section{References}

1. Rayman MP: The importance of selenium to human health. Lancet 356: 233-241, 2000.

2. Ujiie S, Itoh Y and Kikuchi H: Serum selenium contents and the risk of cancer. Gan To Kagaku Ryoho 25: 1891-1897, 1998.

3. Clark LC, Combs GF Jr and Turnbull BW: Effects of selenium supplementation for cancer prevention in patients with carcinoma of the skin. A randomized controlled trial. Nutritional Prevention of Cancer Study Group. JAMA 276: 1957$1963,1996$.

4. Zhou N, Xiao H, Li TK, Nur-E-Kamal A and Liu LF: DNA damage-mediated apoptosis induced by selenium compounds. J Biol Chem 278: 29532-29537, 2003.

5. Lu J, Kaeck M, Jiang C, Wilson AC and Thompson HJ: Selenite induction of DNA strand breaks and apoptosis in mouse leukemic L1210 cells. Biochem Pharmacol 47: 1531-1535, 1994.

6. Kim YY and Mahan DC: Comparative effects of high dietary levels of organic and inorganic selenium on selenium toxicity of growing-finishing pigs. J Anim Sci 79: 942-948, 2001.

7. Medina D, Thompson H, Ganther H and Ip C: Se-methylselenocysteine: a new compound for chemoprevention of breast cancer. Nutr Cancer 40: 12-17, 2001.

8. Jiang C, Wang Z, Ganther $\mathrm{H}$ and Lu J: Distinct effects of methylseleninic acid versus selenite on apoptosis, cell cycle, and protein kinase pathways in DU145 human prostrate cancer cells. Mol Cancer Ther 1: 1059-1066, 2002.

9. Zheng JP, Zhu SF and Dou Y: The effect of Kangqiang selenium and selenium polysaccharide on cell cycle of livers of rats poisoned with arsenic. China Public Health 16: 999-1001, 2000.

10. Yang M, Wang K, Gao L, Han YT, Lu JF and Zou TT: Exploration for a natural selenium supplement-characterization and bioactivities of Se-containing polysaccharide from galic. J Chin Pharmacol Sci 1: 28-32, 1992.

11. Chen C, Zhang Y, Li Y, Chu H and Xi Y: In vivo and in vitro anti-tumour response of selenium-protein polysaccharide extracted from rich selenium Agaricus blazei. Food Agr Immunol 18: 139-149, 2007.

12. Zaidman BZ, Yassin M, Mahajna J and Wasser SP: Medicinal mushroom modulators of molecular targets as cancer therapeutics. Appl Microbiol Biotechnol 67: 453-468, 2005.

13. De Silva V, Woznichak MM, Burns KL, Grant KB and May SW: Selenium redox cycling in the protective effects of organoselenides against oxidant-induced DNA damage. J Am Chem Soc 126: 2409-2413, 2004

14. Liu F, Ooi VE and Fung MC: Analysis of immunomodulating cytokine mRNAs in the mouse induced by mushroom polysaccharides. Life Sci 64: 1005-1011, 1999. 
15. Shang DJ, Zhang JN, Wen L, Li Y and Cui Q: Preparation, characterization, and antiproliferative activities of the Secontaining polysaccharide SeGLP-2B-1 from Se-enriched Ganoderma lucidum. J Agric Food Chem 57: 7736-7742, 2009.

16. Dubois M, Gilles KA, Hamilton JK, Rebers PA and Smith F Colorimetric method for determination of sugar and related substance. Anal Chem 28: 350-356, 1956.

17. Molrnero A, Morales A and Villwereal A: Gaseous sample introduction for the determination of silicon by ICP-AES. Anal Chem 358: 599-603, 1997.

18. Mosmann T: Rapid colorimetric assay for cellular growth and survival: application to proliferation and cytotoxicity assays. J Immunol Methods 65: 55-63, 1983.

19. Bradford MM: A rapid and sensitive method for the quantitation of microgram quantities of protein utilizing the principle of protein-dye binding. Anal Biochem 72: 248-254, 1976.

20. Sun SY: Apoptosis induction by chemopreventive agents. Drug News Perspect 14: 75-80, 2001.

21. Sinha R and El-Bayoumy K: Apoptosis is a critical cellular event in cancer chemoprevention and chemotherapy by selenium compounds. Curr Cancer Drug Targets 4: 13-28, 2004.
22. Zhang ZW, Wei HL, Su HX, Liu JM and Hao CY: Kappaselenocarrageenan induced apoptosis of multidrug-resistant human leukemia cell and its mechanism. Chin J Clin Pharmacol Ther 10: 505-508, 2005.

23. Chen $\mathrm{T}$ and Wong Y: In vitro antioxidant and antiproliferative activities of selenium-containing phycocyanin from seleniumenriched Spirulina platensis. J Agric Food Chem 56: 43524358,2008 .

24. Vermeulen K, van Bockstaele DR and Berneman ZN: Apoptosis: mechanisms and relevance in cancer. Ann Hematol 84: 627-639, 2005.

25. Creagh EM, Conroy H and Martin SJ: Caspase activation pathways in apoptosis and immunity. Immunol Rev 193: 10-21, 2003.

26. Green DR: Overview: apoptotic signaling pathways in the immune system. Immunol Rev 193: 5-9, 2003.

27. Gurp MV, Festjens N, Loo GV, Saelens X and Vandenabeele P: Mitochondrial intermembrane proteins in cell death. Biochem Biophys Res Commun 304: 487-497, 2003. 